\title{
Post-Pandemic Home Design Adaptations: Lessons Learnt for Future Theory and Practice
}

\author{
Mennat-Allah El-Husseiny \\ Department of Architecture, Faculty of Engineering, Cairo University, Egypt
}

Received March 14, 2021; Revised May 28, 2021; Accepted October 21, 2021

\section{Cite This Paper in the following Citation Styles}

(a): [1] Mennat-Allah El-Husseiny, "Post-Pandemic Home Design Adaptations: Lessons Learnt for Future Theory and Practice," Civil Engineering and Architecture, Vol. 9, No. 7, pp. 2542 - 2555, 2021. DOI: 10.13189/cea.2021.090737.

(b): Mennat-Allah El-Husseiny (2021). Post-Pandemic Home Design Adaptations: Lessons Learnt for Future Theory and Practice. Civil Engineering and Architecture, 9(7), 2542 - 2555. DOI: 10.13189/cea.2021.090737.

Copyright $\bigcirc 2021$ by author, all rights reserved. Author agrees that this article remains permanently open access under the terms of the Creative Commons Attribution License 4.0 International License

\begin{abstract}
During the widespread of the Covid-19 pandemic in the year 2020, "staying home" became one of the crucial means of survival. Being an architect and educator during this crucial time raised many questions of whether our current practice and pedagogy place homes in their adequate socio-psychological status. Thus, this research aims to document, analyze and compare selected examples of home adaptations in 2020. The methodology adopted in the research depended on deductive qualitative methods. Analysis of the spaces was conducted by the researcher based on first-hand data. Surveys were distributed among a wide sample, among whom were the in-depth study sample. And finally, interviews were conducted to assess the level of comfort and tools of adaptations made by the residents during the lockdown. The study concludes with lessons learned from the pandemic outbreak to enhance architectural practice as well as pedagogy while addressing the future of home designs.
\end{abstract}

Keywords Post-Pandemic Typologies, Architectural Design, Home Design, Built Environment, Post-Pandemic Pedagogy

\section{Introduction: Theoretical Review on Post-pandemic Architectural Solutions and Understanding the Needs for New Homes Design as a Result of COVID-19}

During the lockdown occurring at the start of the year
2020, due to the spread of the Covid-19 pandemic, "staying home" became one of the crucial means of survival. The home entity was subjected to a sudden transformation from merely a place for shelter and familiar family private and semi-private activities to a place expected to foster and enhance education, recreation, act as a work environment, and provide specialized medical care if needed. Being an architect and educator during this crucial time affecting the globe raised many questions of whether our current practice and pedagogy place homes in their adequate socio-psychological status. Thus, the research aims to document, analyze and compare selected examples of home adaptations in reaction to the pandemic outbreak in 2020. The comparative analysis aims are to answer questions of whether the instant adaptions helped families to find psychological well-being at their homes. The aspects to be analyzed include; adequacy of using homes as workplaces, privacy aspects, possibility of implementing recreational activities, adaptability, and social interaction with neighbors. The study aims to provide lessons learned from the pandemic outbreak to enhance architectural practice as well as pedagogy while addressing the future of home designs in our communities. The methodology adopted in the research depends primarily on deductive qualitative methods. Analysis of the spaces was conducted by the researcher based on first-hand data gathered from residents. Surveys have been distributed among a wide sample, among whom were the in-depth study sample. And finally, interviews were conducted to assess the level of comfort and tools of adaptations made by the residents during the lockdown. The structure of the study is presented in the figure 1 . 
The entire world suddenly faced the same situation resulting from the massive spreading of COVID-19 virus leading to increased death rates and major infections, only after few months of the start of the year 2020. The procedures of prevention and healing were not as effective as expected, leading the world community to apply more or less the same strategies of social distancing, with lockdowns being applied even up till the moment of writing this research. Calls have been announced for what has been named the "new normal", adhering to the uncertainty of the global situation. Sharifi et al [1] explain that it was not unprecedented in humanity recorded history when epidemics reflected on how communities reacted in everyday life, however, there isn't sufficient documentation on how communities and epidemics were effected before the wide spread of the COVID-19 in specific. This is one of the main driving forces in this research, since the outbreak of the pandemic resulted in global attention to shared aspects related to the well-being of all individuals, without any exceptions nor segregations.

Based on Megahed et al [2], the brief explanation of the viral disease spread and precautions are stemming from a relationship between COVID-19 infection and air pollution exposure especially in crowded areas and public facilities. Research proved the correlation between the transition of the virus and the infection through inhalation of a considerable virus load through the person's respiratory system. This is the reason why many countries around the globe have applied strict lockdowns, quarantine, and social distancing to limit the spread of the virus which was both highly spreadable and leading to dangerous infections which result in increased death rates, [2]. However, some of the problems associated with the pandemic, as Schnitzer et al. [3] highlight, result from isolation which may lead to psychological and social problems and have huge impacts on the world's financial and economic stability from another side.

Based on the arguments of Sharifi et al [1], the effect of the pandemic on social issues has been discussed in developing and under-developed countries equally since the contagious effect is the same for everyone, taking into account the viral infection rate spread in every country. The focus of most research pieces was on the negative impacts of the spread of the virus, but there were also debates regarding the possible positive effects resulting by the current situation. Also, some debates have been raised regarding the unequal opportunities of survival between the different communities as a result for economic inequalities. And this debate holds roots in historic incidents of pandemics highly affecting under-developed communities due to decreased awareness or decreased opportunities for enhancing and maintain public health. However, with the claim of architectural solutions which help in reducing the virus load in the indoor built environment and decreasing the possibility of infections, it became extremely important to regard matters of affordability and efficient solutions to solve some drawbacks of the pandemic. Accumulation of indoor air pollutants contributes to a great extent to what is known as "sick building syndrome", as has been researched and studied previously. Thus, users of buildings in a healthier setting are more susceptible to enjoy enhanced health environments, as claimed by Sharifi et al [1].

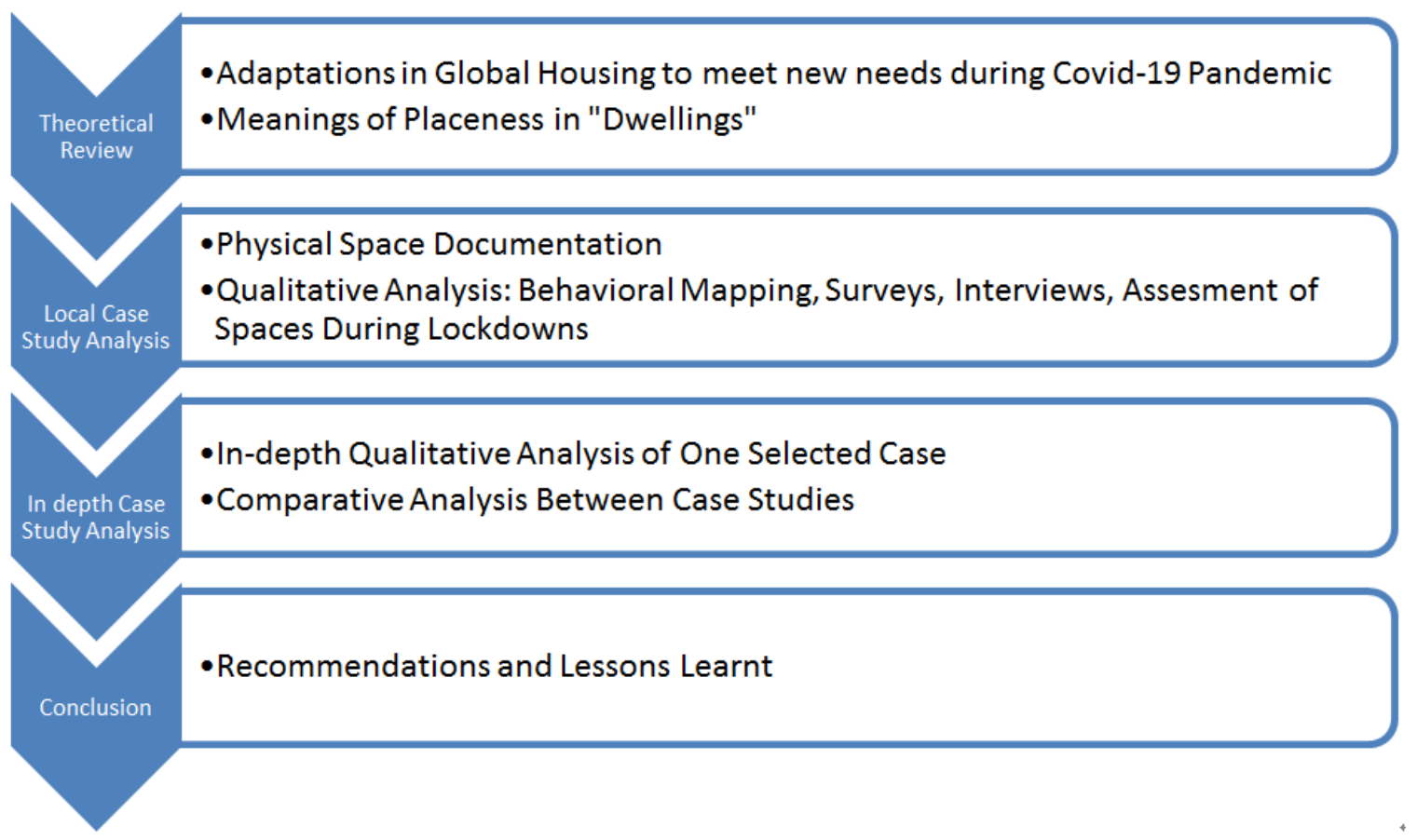

Figure 1. Structure of the Study (Developed by Author) 
This results in an important urge to face the pandemic on both micro-scale and macro-scale levels. Based on hazard elimination concepts, the possibility of getting exposed to Covid-19 can be reduced through engineering controls. This can be implemented through providing better indoor environment. Part of this relies upon the affordable re-adaptation of buildings design and operation systems to provide more wellness in buildings, both physically and psychologically, [1].

In addition to this, it has to be taken into account that it is important to apply better designs focusing on humanistic well-being. This aims to limit or totally reduce the negative impact of "sick buildings syndrome" on the health of its users and residents. This leads architects and designers to work on protecting the users and dwellers, as an ongoing sustainable model which aims to avoid future health based pandemics, [1].

It can be summarized that up till the time of writing this paper, the research on COVID-19 and its association with architecture and urban design have been grouped into four main categories [1];

1. Environmental quality issues,

2. Various socio-economic impacts on users,

3. Management and governance,

4. And finally the role of transportation based on urban design strategies

Thus, relevant to the scope of this research, the upcoming part will focus on the socio-economic impacts of the lockdown and social distancing on home dwellers precisely. It has been observed that pandemics may be an opportunity to enhance the quality of life in cities by paying more attention on social behavior through health perspective in planning and design [4]. Social behavior is considered a key factor in dealing with this pandemic in the current era. Per that, El-Helou [5] has previously observed that one of the main healing cities attributes is common and related to the micro as well as macro-scale design. This both affects physical health and mental health over time.

As has been asserted and researched by several scholars, the highly contagious COVID-19 virus leads to a serious global health problem, which is being reflected on various levels through the serious social distancing measures parallelizing major events and social gatherings world-wide, like for instance; Tokyo Olympics, Festivals and Pilgrimage trips. Several debates regarding the patterns of the person in space and the foreseen intensive utilization of information technology had led to the social and institutional acceptability of 'new normal', due to the strict need for social distancing, [6].

Among but not limited to ideologies and concepts related to place-making and proxemics need to be revisited especially as related to home designs in middle-income classes, relevant to what has been discussed in the previous part [6].

Thus, the upcoming part will expose major aspects to be taken into account while upgrading or re-visiting the designs of homes, post the current era pandemic. This is not only crucially important to facing the current situation with all its draw-backs but most importantly, to be ready for possible expected waves of viruses. The first aspect related to place-making in home design is place attachment, which is "highly influenced by an individual and his or her personal experiences" [7]. It is diverse and highly dependent on an inter-relation between the person's habits, in addition to their experiences [8]. Accordingly, it is strongly in accordance with the person's perception and lifestyle patterns. "Places of attachment" are resembled but not limited to "the home", neighborhood, certain public spaces, and landscapes interacting with nature. The degree of person's attachment to these places is measured by several specific qualities which depend on the nature of the facility and how users interact in the space. As scholars group the qualities, they are summarized to include: "aesthetics, heritage, family connection, recreation, therapeutic, biological diversity, wilderness, home, intrinsic, spiritual, economic, life-sustaining, learning, and future". Thus, it has been claimed that place attachment after Covid-19 pandemic should provide re-assessing several of the spatial qualities with more focus to aspects of healthier and more healing environments. This may also lead to the rise of the needed study of the post-disaster traumatic influences, according to Salama [6], "which aims to examine the relationship between a city, urban area, or neighborhood and their inhabitants' attitudes and emotions".

It has been argued that personal space measurements in addition to studies of proxemics theories explained in the mid-1960s [9] are important to re--visit in the context of social distancing. Personal space means how a person interacts intimately and can be visualized by a bubble which had invisible limits surrounding the person's body into which strangers are not allowed to come through. This specific imaginary bubble moves with person everywhere. Based on empirical studies; Hall [9] pinpoints the distances relatively in accordance with the personal space which is likely to be applied on the distances between different people depending on their relationship to each other. This results in the four well-known distance ranges; intimate, approximately 1 $46 \mathrm{~cm}$, personal; approximately $46-122 \mathrm{~cm}$, social approximately $1.2-3.7 \mathrm{~m}$, and finally the public $3.7-7.6$ $\mathrm{m}$. Based on that, Salama [6] exposes that "social distancing measures and the minimum allowance of 2 meters personal distance, the relative distance ranges would entirely change".

According to Donohoe [10], although proxemics and spatial relationships between individuals have range variations depending on culture and context, there are two aspects which are in common between all founding constants principles of spatial organization and experience, "the posture and structure of the human body, and the 
relations (whether close or distant) between human beings". Meaning that body and place are inseparable.

Those debates recall what Bachelard [11] advocates for the home to represent, "protected intimacy" constituting the aspirations and the realities. He describes it simply as "inhabited space". But it is also more than that for Bachelard [11]. He recognizes that the dwelling place of home is intimately connected to memory. Finally, Coolen et al [12] debate our understanding of the perceptions of the houses, homes and dwellings by exposing five factors which highlights the differences between those terminologies. For instance, "home" defines the material entity, while dwelling relies on the activities and patterns related to activities and social attributes of the spaces.

From another side, digging deeper into structures of human dwelling, importantly less advantaged clusters, Lai et al. [13] present that the condensation of population and density in informal settlements of the less fortunate population are the real challenge to be faced. Social distancing in self-structured settlements has thus far been difficult to implement as a result of overcrowding, [13].

Lai et al [13] also expose that attention should be paid to micro-level housing attributes. Among those are hygienic and natural ventilation attributes, which play an important role in limiting the spread of viruses. Future housing needs to take into account the presence of spaces of multiple functional spaces. Those spaces would allow the necessities of new patterns of working from home and the sort, which constitute the new normal after Covid-19 pandemic [13], among other solutions as will be explained in the analytical part.

Eltarabily et al [4] explain that it is currently the era that is the beginning of the momentum of dynamics in values and habits inside our homes. From social behavior view and after self-isolation it can be evidenced that stacking multiple residents in apartments buildings where spaces for isolation and hygienic limitations ought not to be the housing best options. Also, from the public health vision isolating in separate houses is more adequate than in apartments. These findings are identical to the results of many studies that examined the increase health hazards whether physical or psychological in comparing apartments in stacked buildings with houses [4].

Eltarabily et al [4] further explain that considering the social behavior point of view nowadays dwellers tend to invest a larger portion of their time at indoor environments, especially during lockdown. Accordingly, the homes they dwell in affects their health directly. House closures and isolation measures have transformed the original functions of houses into places to sleep, work and entertain [4].

Moving forward to the notion of hybrid space, which is currently emerging as a need in post-pandemic home designs, Ali [14] explains that hybrid space is multi-layered, depending on its purpose, more specifically in the sense of technological milieu. This means an area which involves information that flows within it; it is an objective synonym for the virtual space. Foucault describes the current age as the age of space. He referred to Bachelard's [11] phenomenological viewpoint about lived space to describe this kind of heterogeneous space, "we are living not in a homogeneous and empty space but, on the contrary, in a space that is laden with qualities, a space that may also be haunted by fantasy." [15]

Finally, Tokazhanov et al [16] summarize that to ensure resilience and sustainability, future homes should be able to cover the phenomenological questions resulting from the current global pandemic, of whether our built environment can decrease the spread of diseases [17], and how to create better indoor environments, and how to enhance the physical and psychological well-being of long period's home dwellers. Stemming from that, it can be summarized that the basic needs for households are natural illumination, adequate natural ventilation and enhanced air quality, in addition to adequate acoustics levels which are manifested in various modes. Also, comfortable climatic conditions in the micro-scale environment. Additionally, those aspects represent the criteria of analysis in the case studies selected.

\section{Materials and Methods of Analyzing Homes Adaptations to Meet Lockdown Needs in Cairo}

Based on a qualitative descriptive study for six homes in Greater Cairo, the next part would highlight the changes, adaptations, and level of satisfaction for homes' dwellers during the first lockdown, which occurred during the period of March 2020 up till September of the same year. The limitation is the number of case studies which managed to provide before and after alterations. The constraints were the spontaneous situation, and the limited modifications or adaptations possible to take place due to the non-preparedness of the users to the sudden wide-spread of the pandemic. This lockdown was a sudden one due to the outbreak of the COVID-19 virus in Egypt, resulting in pause in all types of social interactions, face-to-face learning, and reduced office space usage in official workplaces, and "stay at home" continuous calls. The average documented cases of infection in Egypt during the peak of the virus spread curve were 10,000 cases per day. Although not considered a big number relative to other countries, however, death rates reached $60 \%$ during the peak time, which was higher than the average of what was documented for other countries in the region.

Constraints which faced home-dwellers of the study were mainly that the first lockdown was sudden, without prior expectations nor precedents for the situation. Most activities of working, educating, recreating in addition to other normal dwelling activities had to be catered for at 
homes. Most homes required adaptations and minor or major changes to face the sudden lockdown, based on the possibility of the available infra-structure of homes, the cost and the rapidness of application. Aside from the social and utilitarian requirements, psychological and proximity considerations needed to be re-questioned. In addition to this, health and hygiene aspects needed to be also taken into account, including quality of ventilation, sun-light exposure, and the possibility of sanitization, and the possibility of finding an adequate isolation room, in case a family member was affected by the virus. This is of course relevant to the number of dwellers of the home in relevance to its net area.

Data collected in the upcoming analysis was gathered as first-hand information from architecture students [18], whose daily activities altered completely during the course of the quarantine. The students themselves were subjected to the sudden transitions, and this lockdown took place during the course of their academic semester. This required the young architect to create alterations in their own homes, to fit and survive the lockdown situation. Depending on the nature of each family, the most dominant aspect which needed focus was altered. Accordingly, the upcoming part will discuss the nature of the changes; based on activity mapping before and after the start of the lockdown, each case varies according to the nature of the challenges.

The first case Fig.2, showcases a family consisting of four members, two of whom were still in their education years. The family room space was transformed into a studying-from-home zone, as indicated in the figure below since the bedrooms were too small to accommodate any other activities other than sleeping. The reception zone, usually in Egyptian culture preserved for important guests, was used for family gathering activities. And furniture layouts were adjusted, to create an enclosure for the interactive space, which helps in combating the drawbacks of the lack of socialization and to provide better psychologically soothing space.

The second challenge which needed to be taken into account, in this case, was the issue of providing an extended recreational zone. The balconies were utilized for providing mini-gardens, which were also used as a buffer zone to reduce stress, provide fresh air while maintaining "stay at home" calls. The home users' satisfaction increased from medium to high after applying those minor adaptations, based on surveys related to physical, mental, and psychological comfort.

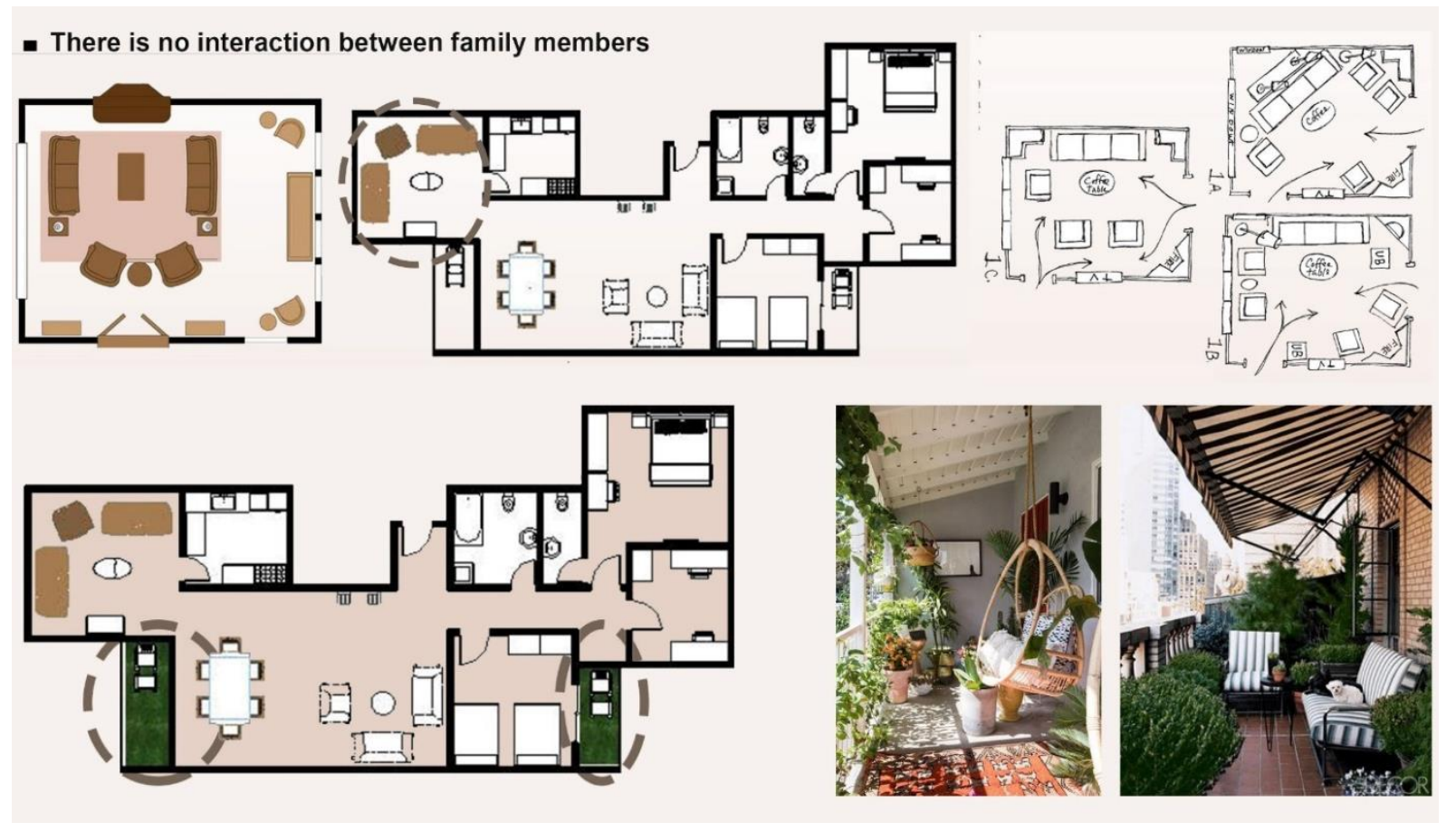

Figure 2. Case Study One, (El-husseiny, 2021). 

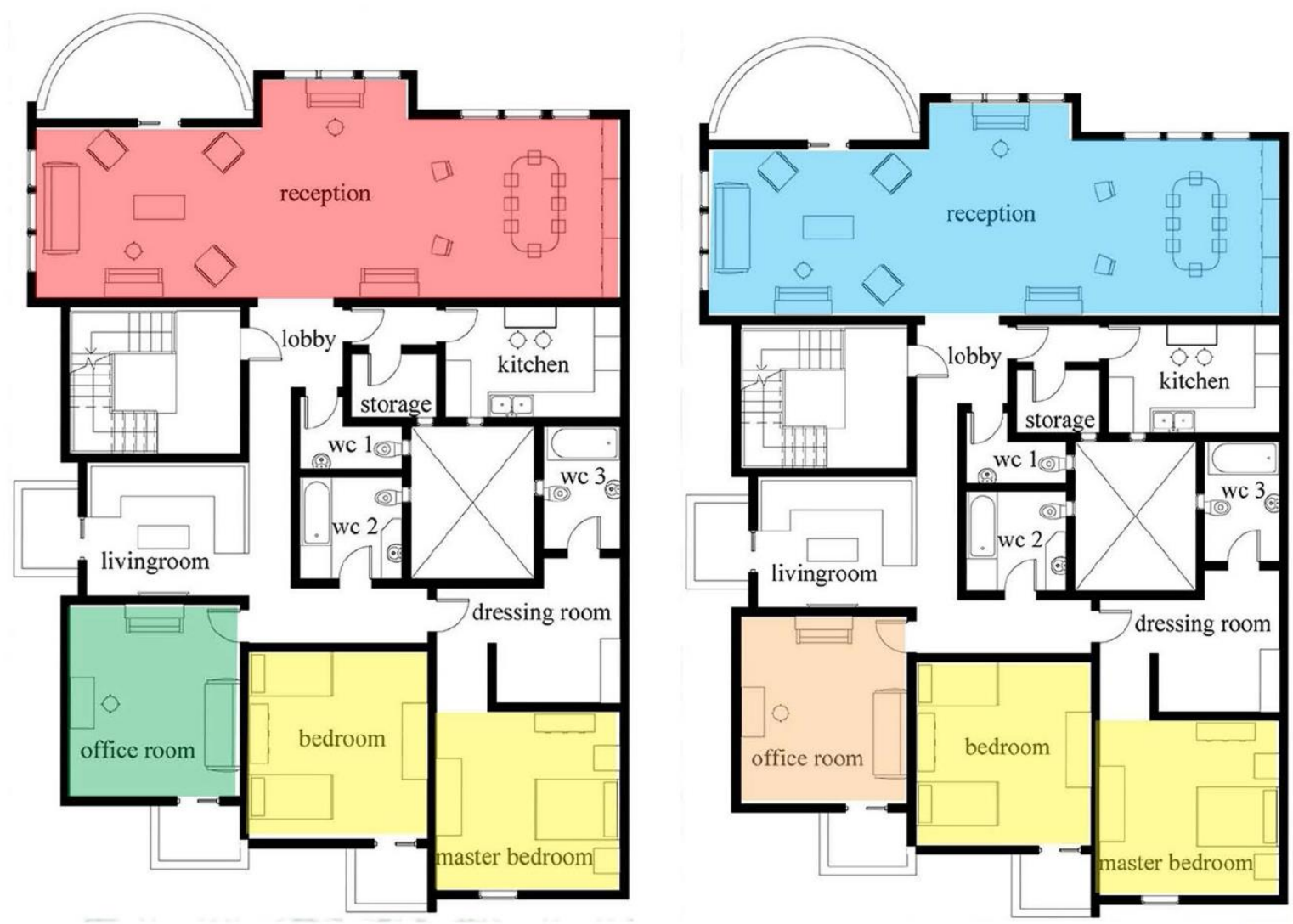

Figure 3. Case Study Two, (El-husseiny, 2021).

The second case, shown in Fig.3, which has been studied qualitatively before and after the lockdown featured a special case since the lord of the family was a musician who used to practice and perform at remote studios. However, as seen in the mapping of activities below, during the lockdown resulting from the outbreak of coronavirus, he had to practice and record for online recordings using the area designated previously for receiving guests. As claimed by the young architect who was interviewed for this case, being a student along with his sibling, demanded that music practice and recording be as far away as possible; zoning-wise from the bedrooms, which were used for studying and sleeping as well. The office room previously used merely for paperwork was altered to accommodate sleeping as well, since two siblings sharing one room was not very practical, given that both needed to attend online sessions while maintaining privacy. Finally, the student explained that his family was considered a fortunate one, to have enough and adequate spaces, which were slightly altered. It was possible to accommodate the needs of all the family members who were all locked down during the first quarantine.
The third case to be addressed here which is relevant to our study was a case of a student of architecture, whose home was a two levels condo, located in Giza. The student reflected that his home design did not cause any zoning issues even when all family members needed to stay at home during the first lockdown. The studying/sleeping activities were well provided, which allowed the family members to select better micro-climatic conditions in the Northern and Sothern orientations where the home facades were oriented. During winter, which consumed a period of two months of the lockdown, the reception initially designated for receiving guests was utilized by family members to enjoy the warmth and natural light. They also believed that this created better hygienic conditions. During spring and summer, when the pandemic effect was slightly reduced, however using outdoor recreational facilities was still limited. Family members used the Northern parts to introduce natural ventilation. This activity was also believed to have reduced the risks of airborne disease. All those studies are explained using the mapping of activities and climatic conditions on the home design as in the illustrations below, in Fig. 4. 


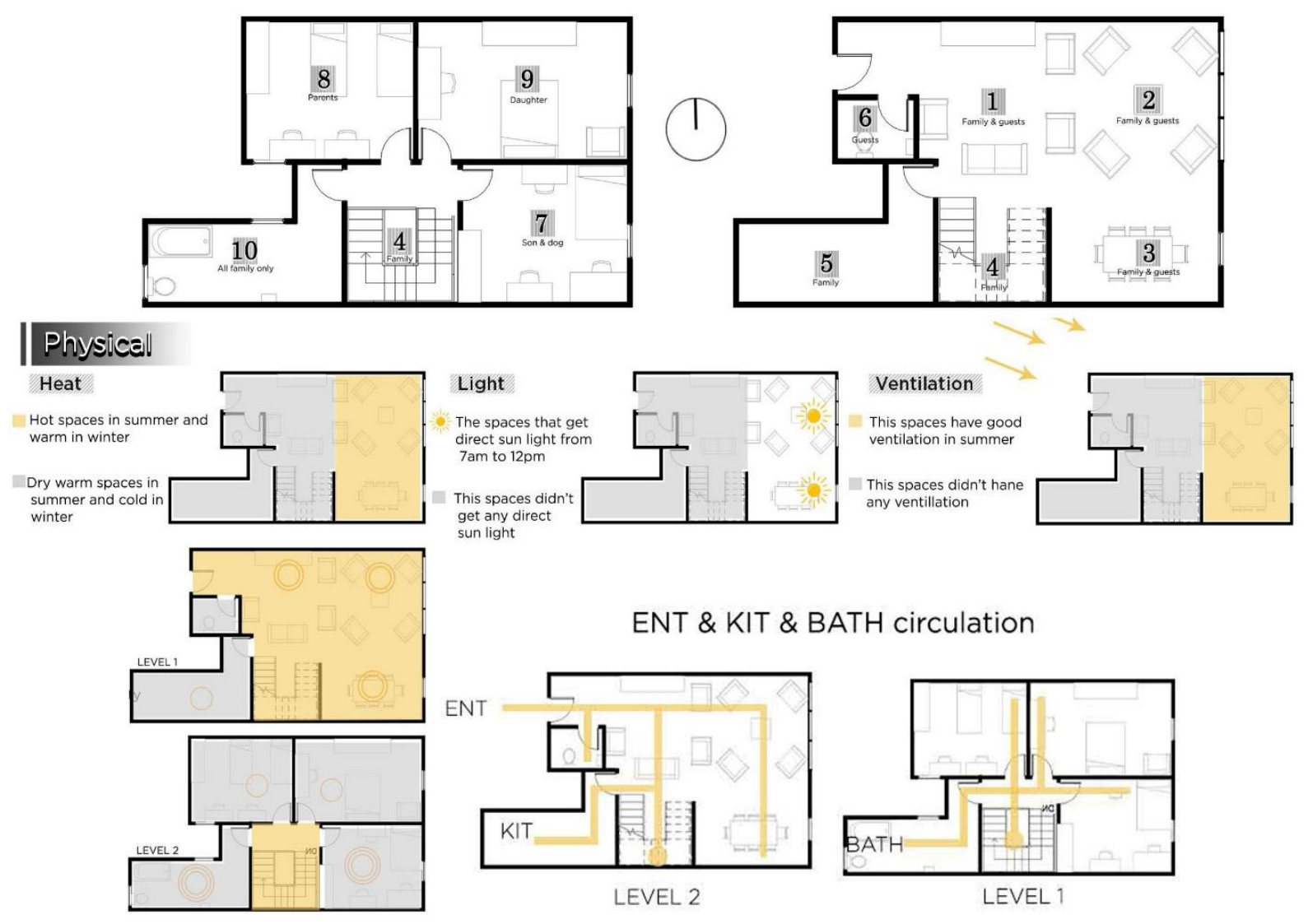

Figure 4. Case Study Three, (El-husseiny, 2021)

The fourth case analyzed below in Fig. 5, showcased a major issue of the sudden transformation for working from home. The home area relatively appropriate for the existing family members during normal circumstances was not adequate for the new normal, precisely in aspects related to providing home workspaces for each family member. Thus, the major alteration which took place as shown in the figure below was to compromise part of the family living zone, to create a suitable workspace for one of the siblings. This enabled the other sibling to use the private bedroom originally shared by both, to attend online classes or accomplish his studies. Although this compromised the space utilized for socializing between the family members, which was re-adjusted as to furniture allocations, however for long hours of work at home, this created also a possibility for establishing a better sense of place. This was believed to strengthen family bonds based on the users' point of view, instead of having all workspaces segregated in islands at the same home. Also, this affected the family long term plans to provide more space in the living area for shared workspaces, at times which did not require much privacy. This enabled the conversion of the family gathering space into a co-working zone. This was highly appreciated as being the heart of the house where vital activities such as cooking and dining usually took place. 


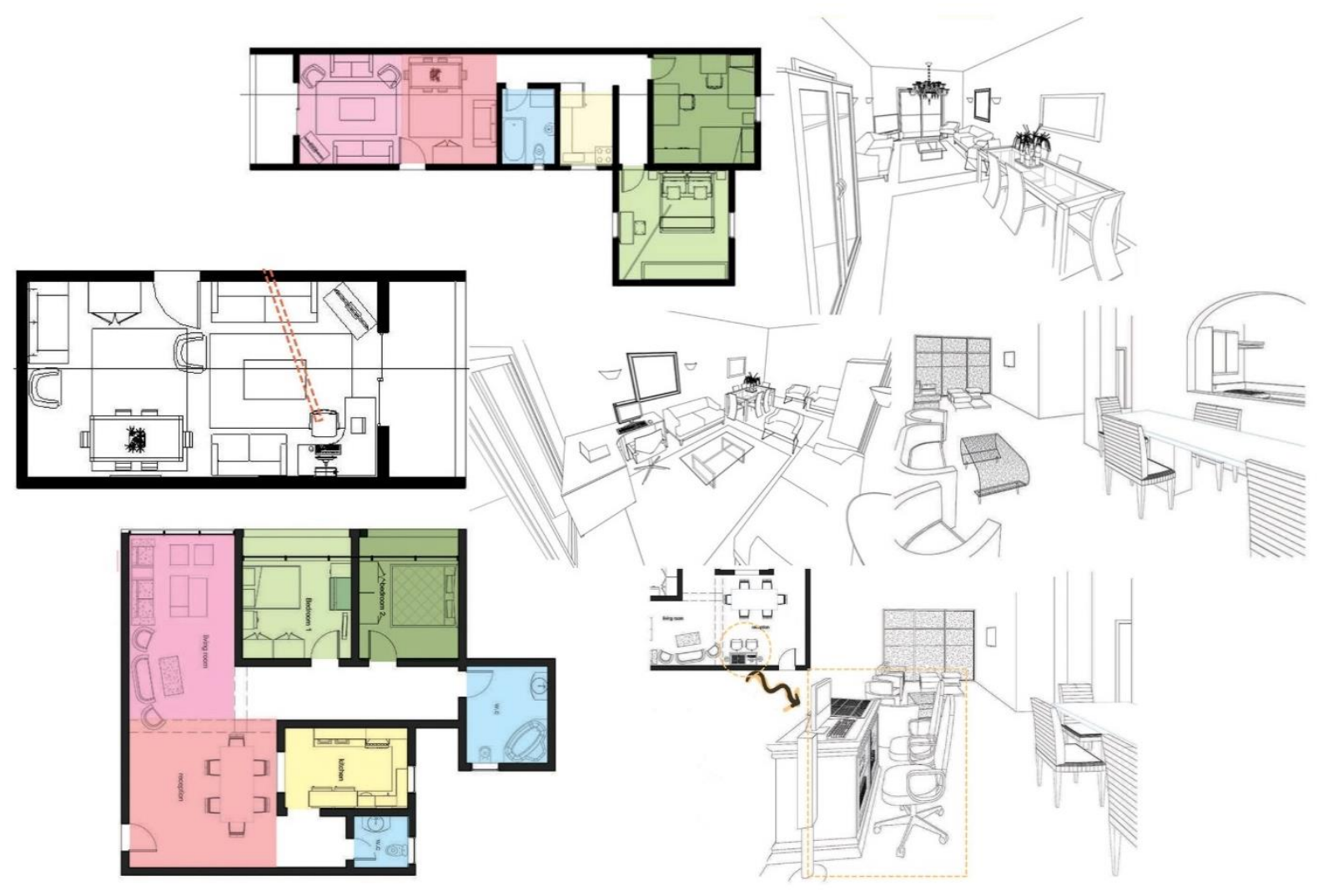

Figure 5. Case Study Four, (El-husseiny, 2021).

The last case in the scope of the analysis was conducted qualitatively in the assessment of the needed transformations of Cairene homes during the quarantine forced by the spread of the COVID-19 virus, witnessed a different pattern of uses. The family members were four siblings and a single mother, who shared a two bedrooms apartment. The main challenge which needed to be solved was the day-long multiple calls and sessions the four siblings had to run. The challenge which faced the student implementing the study was the fact of being the only male in the family, which forced him to maintain the privacy of his family members from being exposed online, especially in a conservative background as was the case of the family being studied. The solution was to provide the student with a separate room, and two female siblings would share one room, while the mother and the third sibling would adapt personal spaces in the guests' reception room. This case, analyzed below in (Fig.6), managed to although adapt the lockdown of five family members in a relatively tight spatial distribution, was still fortunate enough as shown below, to be able to attain some sort of personal spaces. However, in other cases, which have been interviewed, yet were not able to showcase their homes for the study, major problems were present, such as the total lack of natural ventilation in some rooms, crowdedness of rooms up till sharing four family members the same physical space, the need to use a shared bathroom with other housing units, and the inability to attain stable affordable internet connection at home, accordingly, some needed to attend online classes from friends' homes if this was allowable. All those issues highlight the limitations of this research, which only focused on problems associated with usages, social patterns, and different layers of comfort, in dwellings of middle-income classes. 


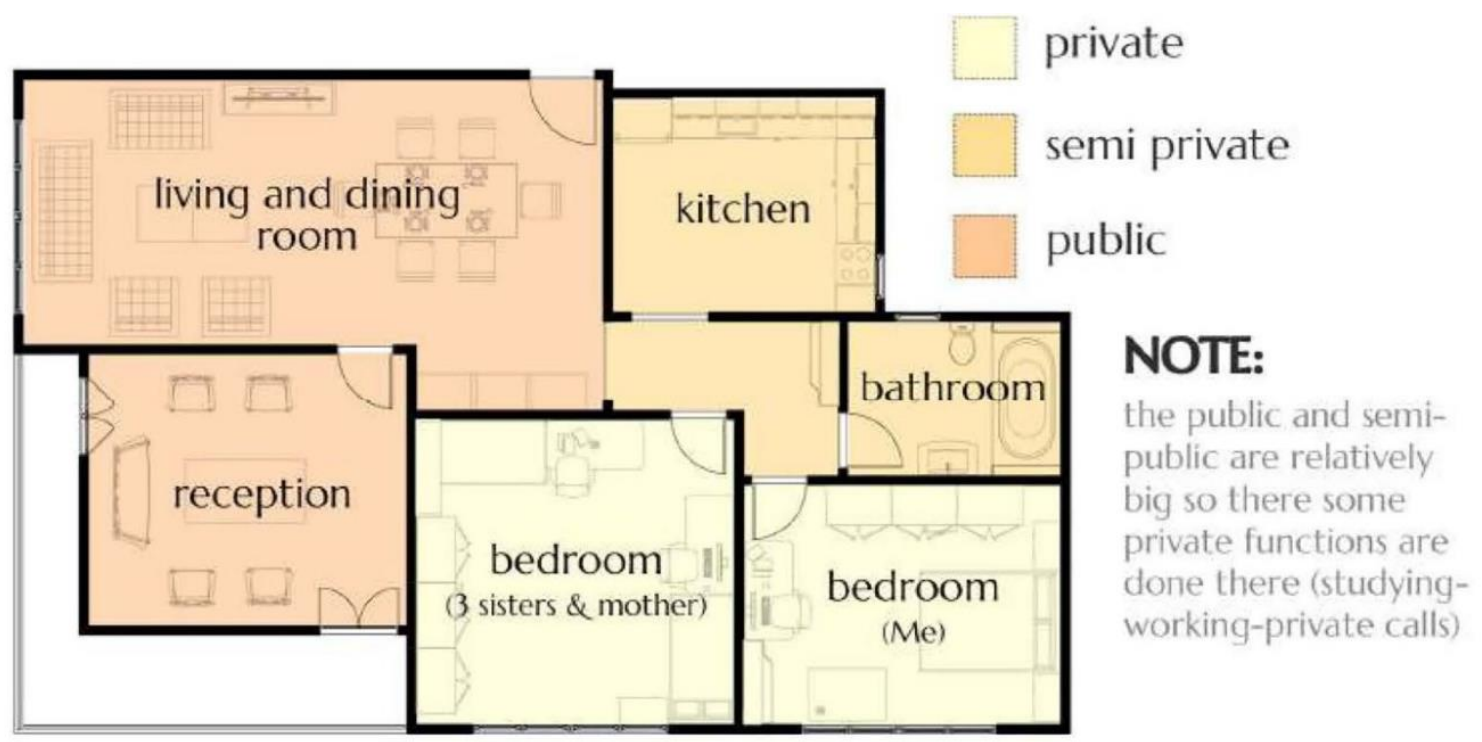

BEFORE QUARANTINE

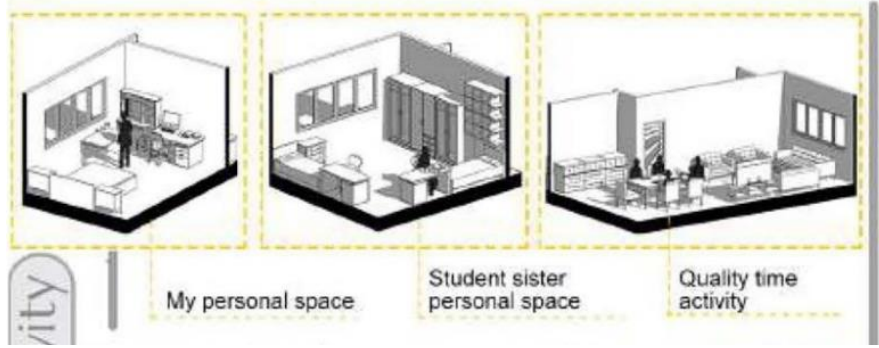

AFTER QUARANTINE

Figure 6. Case Study Five, (El-husseiny, 2021)

The following in-depth qualitative analysis focuses on a comprehensive study for a residential unit, with a high middle-income class family prototype in Cairo, (Fig. 7). This case study aims to shed light on how families who were fortunate enough to attain a tailored design home were able to adapt to the lockdown, on several levels to be explained in the analysis. The criteria of analysis were to evaluate privacy issues, work from home, study from home, the possibility of self-isolation in case of infection, and lastly, the possibility of maintaining positive psychological health during the lockdown.

The family profile for the studied home consisted of four family members. The parents are architects, one is a practitioner who used to work from the office before the lockdown. The other is an academic who lectures at University. The other two family members were aged 5 and 10. And both attended school regularly, before the lockdown. During the lockdown, the family members had to stay at home for four months, during which, academic studying was functional for three of the family members at home, during the same timings of the day. Regarding privacy issues; as can be seen in the plans analysis below; the first floor was designed to be in relation to the garden and to host guests; in a semi-public mode. However, during the pandemic, it has been transformed into the workspace for the mother, who needed to maintain the online tutoring privacy without distractions from the kids, to be able to print material and meet students virtually, also without interrupting the privacy of other family members. While the garden space has been the sole retreat for the four members, after the end of academic sessions, to gain some energy after the long hours working online.

The second level was designed to be an open plan area for family interaction. However, during the lockdown this did not prove to be an adequate architectural solution. Since the father needed to work in a quiet place, using the office which is open to the living room forced everyone to avoid using the living space. Instead, the bedrooms were adapted to incubate the online sessions for the kids, to maintain the privacy of the family members, while concentrating in the virtual classrooms. In most cases, the spaces were suitable for the adapted usage. In some special times, when there was a case among the family members suspected of being infected and had to be isolated in a room which has a private bathroom, the only available one was the parents' room. Thus, this solved the situation and opened up another perspective for the possibility of providing another in-suite in the boy's room in the third level, to be prepared for a similar situation without forcing major changes. 

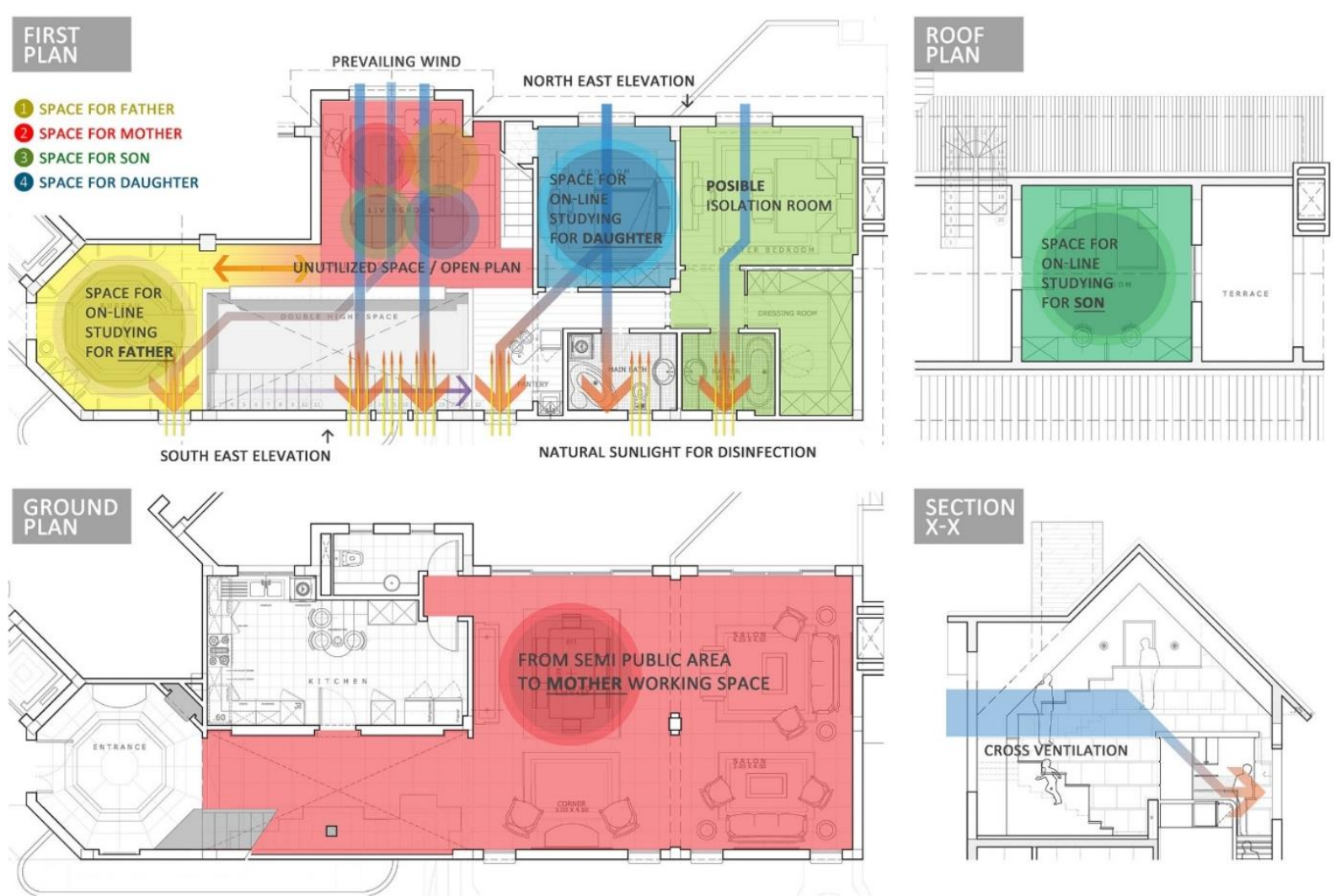

Figure 7. In-depth Study, (El-husseiny, 2021)

In regards to the psychological impacts of the lockdown, most were overcome through the separated yet partially connected bubbles of spaces that were created during the day when online classes were provided. Also, natural light and ventilation helped in this regards, since the orientation for most spaces faced both North West and South East, thus natural ventilation was at its best, also, the spaces were subjected to natural sunlight, which acted as a model of thermal comfort and as well for better hygienic qualities.

The main problems associated with the home design which was not very successful during the lockdown period were related to the effective utilization of the heart of the house. Before the lockdown, it was the living room on the second floor, with the attached kitchenette, or the dining room on the first floor with the main kitchen where most family gatherings, family interactions, and activities took place. Those were vertically also aligned in the design to highlight the importance of the heart of the house. After the experience of the lockdown and based on interviews with the family members, it was concluded that it would have been better if this space could accommodate work capsules or nooks, where the kids can have their online classes in a space that is still semi-private zoning, rather than being secluded in their rooms. Also, it was suggested that the backyard could have been more incorporated with the heart of the house, so that surveillance could be achieved for safety measures over this place if the kids were having a break while their parents were still working from home. Those aspects can be used to guide the next part related to the lessons learned from the analysis of various homes during the lockdown associated with the spread of the COVID-19 virus.

\section{Results of the In-depth Study for Applying Resident's Demands and Alterations During and after Lockdowns}

The following table (Table 1) highlights the main challenges faced by the studied cases and the means of adaptations. This will inform the discussion with recommendations for aspects of enhancing future home designs, which would be also applicable after the end of the current pandemic since it will adapt into what has been commonly known as the new normal to conclude the qualitative analysis concerning users' degree of satisfaction with their home's design during the pandemic lockdown. The survey has been conducted to evaluate and collect as many reflections as possible. Those reflections would help formulate the expected future guidelines, which are not limited to the studied group. The sample of study for the survey was 104 respondents, $85.6 \%$ females and $15.4 \%$ males. The majority of the respondents $(59.6 \%)$ were graduated and working, with an age range of 24-40 years old. Above 40 years old were $9.6 \%$, while $31.7 \%$ were in the range of 18-23 years old. Most respondents 
selected the range of family members sharing the same house ranging from 3-5 members were 76.8\%, 2-1 family members were $12.46 \%$, while $6-7$ were $10.3 \%$. The most important point to be taken into account was that $62 \%$ of the respondents were either working or studying from home during the lockdown. Among the sample studied, $48.1 \%$ did not have a separate room for their use. However, with this taken into account, $57.7 \%$ stated that their privacy was not affected during the quarantine period. Additionally, $39.6 \%$ of the sample taking the survey mentioned that their work needed a separate room. While $66.7 \%$ of those respondents answered that they didn't have the needed space.

Table 1. Adaptations conducted in the six studied cases to meet physical and psychological comfort
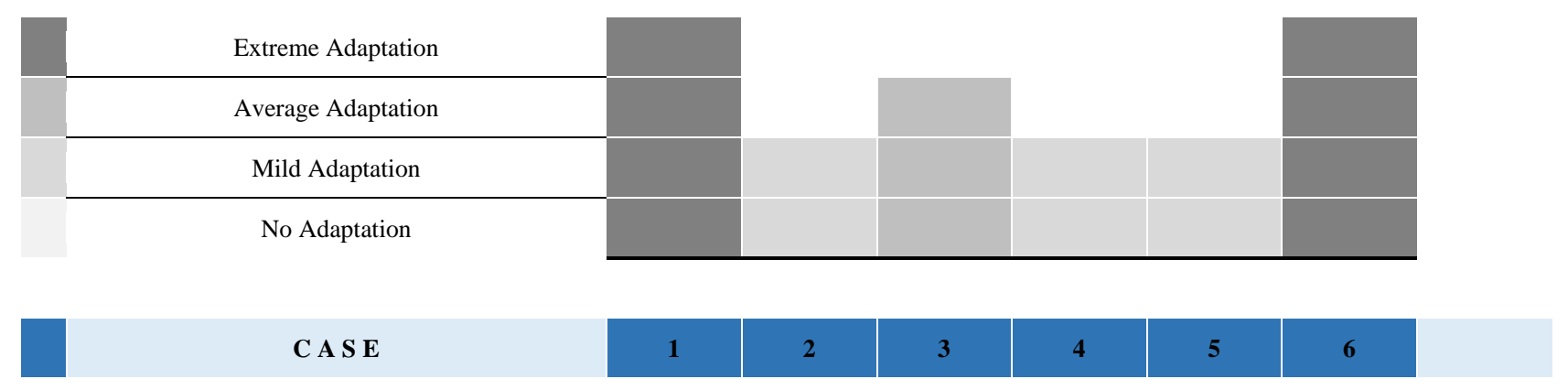

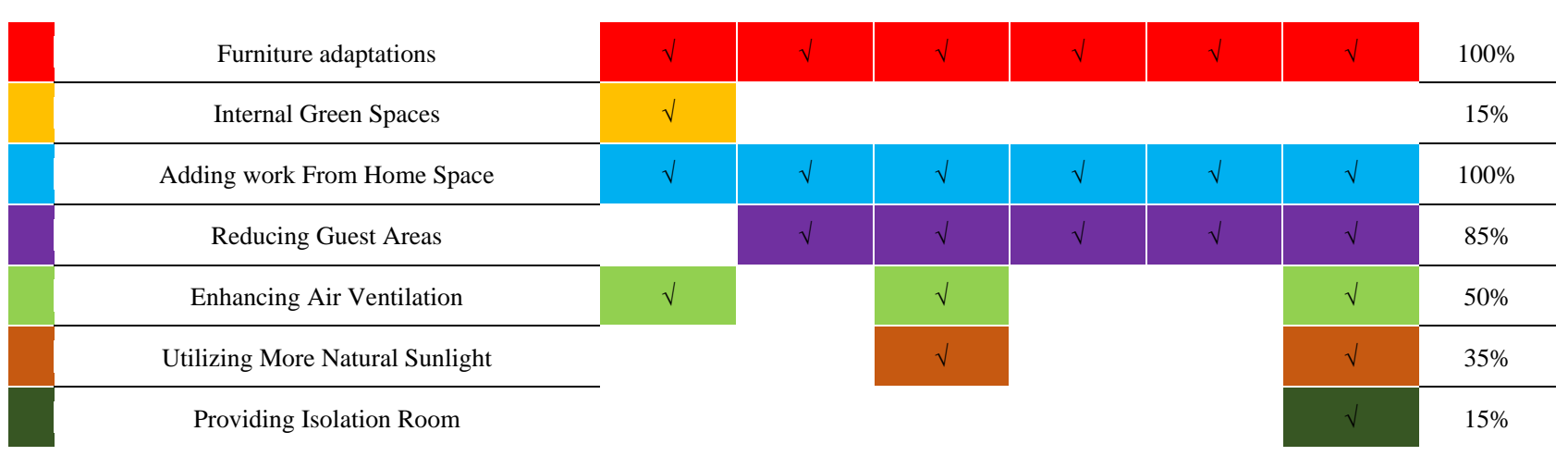




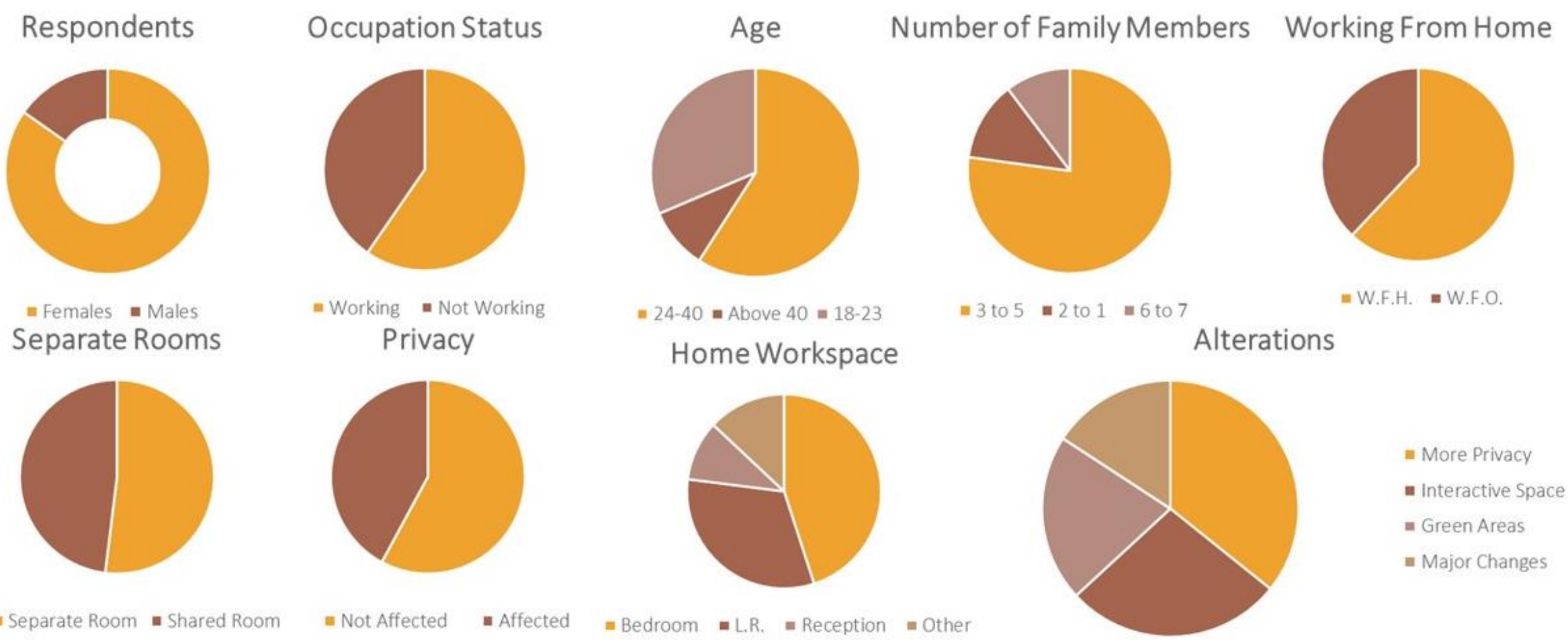

Figure 8. Pie-charts showing the Surveys' Percentages, (El-husseiny, 2021) 
However, it was interesting to observe that from the collective sample, $61.5 \%$ answered that they preferred working from home rather than going to their workplace itself. When asked about the most used space to work from, $45 \%$ of the respondents mentioned that they used their bedrooms, $32 \%$ used the living rooms, $10 \%$ used the reception area while $13 \%$ specified "other" as their work spot. It was an interesting observation though to regard that $52 \%$ found it quite difficult to focus while working from home, which required that they work for extra hours during the day. Also, $50 \%$ of the sample mentioned that their home workspace was adequate, while the other $50 \%$ mentioned that they needed more space, or implemented some organizational and color changes to adapt to staying at home for a long time. Finally, the respondents highlighted their preferences to add more layers for privacy $35.8 \%$, more interactive spaces $27.4 \%$, more green areas $21.1 \%$ and larger areas $15.8 \%$, when questioned regarding the possible alterations to adhere to in the future development or case of selection of a new home. This data will be further reflected upon in the discussion of the research to extract the lessons learned for future home designs. The summary of those percentages are indicated in the figure 8 .

\section{Discussion: Lessons Learned from the Pandemic: Future of Home Designs in Developing Countries between Theory and Practice}

Based on what has been studied qualitatively beforehand, it can be concluded that post-pandemic homes need to apply standards that are different from what they are designed for now. Post-pandemic homes need to abide by the presence of natural ventilation in all spaces to allow for direct sunlight to disinfect vital spaces, provide fewer spaces for guests in favor of adopting recreational, social-interactive, and more psychologically appealing work from home spaces. Those aspects are essential for home designs. However, even if areas are limited, adaptations and quality of initial home designs can provide the future home with its essential needs. Additional aspects which need to be taken into account in cases of higher housing levels, are to provide greenery even if small in area, to provide thresholds for disinfecting users or goods admitted to the house, and to allow for at least one space where a sick family member can be isolated in case this is needed. Accordingly, a successful future home would be one where physical and psychological well-being would be the priority for designers. Those aspects are important for theorists and designers to take into account, while founding standards for futuristic homes.

\section{Conclusions}

Coronavirus pandemic resulted in major milestones in all aspects of science, nevertheless, affected architectural design discipline to a remarkable extent. The research aimed to shed some light on the incubator of humans during lockdowns caused by Coronavirus, which are the home entities. After reviewing the abundant literature relating and analyzing the effects of the pandemic on architecture both on micro and macro scales, qualitative analysis of selected home samples has been conducted based on users' documentation, surveys and interviews. This analysis resulted in aspects to be taken into account while re-thinking the entity of the home in the post-pandemic era. It was proven that all the homes studied qualitatively or through the survey applied from mild to major adaptations on their homes during the pandemic. Adaptations ranged from furniture re-allocation, using guest spaces for other activities, adding work from home spaces, providing more privacy for various dweller especially if more than one family member has been working from home, and finally, dedicating a room for isolation when needed.

Those aspects foresee the future of home design. Futuristic homes need to be more sensitive to proxemics and privacy aspects of family members dwelling in the same home. Natural ventilation and sunlight are to be more wisely adapted for more healthy home environments. Workspaces would be more prioritized than guest zones, especially in education and in more oriented technological disciplines. Internal recreational areas would also be taken into account in the shadows of the lockdowns experienced during the pandemic. Those new patterns can be further investigated when the pandemic is over, in order to asses to what extent the design of the home can sustain a comprehensive and inclusive mode of living in a changing era. However, the study proved that since adaptations have been implemented spontaneously by dwellers, the patterns studied would be better taken into account for future home design.

In addition to this, flexible home layouts, and adaptable spaces for multi-purpose uses can be a solution, which can be implemented through new technologies in adaptable furniture and in applying light weight building materials which can be re-adjusted according to emergency needs.

\section{Acknowledgements}

We are very grateful to the Environmental and Behavioral Studies course staff and students at Cairo University for providing us with the raw material and accepting to undertake the surveys which enhanced the study enormously. 


\section{REFERENCES}

[1] A., Sharifi, A. R Khavarian-Garmsir, The COVID-19 pandemic: Impacts on cities and major lessons for urban planning, design, and management. Science of The Total Environment, No. 749, 2020. https://doi.org/10.1016/j.scit otenv.2020.142391

[2] N. Megahed, E. Ghoneim, Indoor Air Quality: Rethinking rules of building design strategies in post-pandemic architecture. Environmental Research, No. 193, pp.2-9,2020. https://doi.org/10.1016/j.envres.2020.110471

[3] M. Schnitzer, S.E. Schöttla, M. Koppa, , M. Barthab, COVID-19 stay-at-home order in Tyrol, Austria: sports and exercise behaviour in change? Public Health, No.185, pp.218-220,2020, https://doi.org/10.1016/j.puhe.2020.06.0 42

[4] S. Eltarabily, D. Elghezanwy, Post-Pandemic Cities - The Impact of COVID-19 on Cities and Urban Design, Architecture Research, Vol. 10 No. 3, pp. 75-84. , 2020. https://doi:10.5923/j.arch.20201003.02

[5] M. El-helou, Shaping the City that Decreases Overweight and Obesity through Healthy Built Environment, Journal of Contemporary Urban Affairs, Vol 3 No. 2, pp. 16-27, 2019. https://doi:10.25034/ijcua.2018.4697

[6] A. Salama, Coronavirus questions that will not go away: interrogating urban and socio-spatial implications of COVID-19 measures, Emerald Open Research, Vol. 2, No.14, 2020. https://doi.org/10.35241/emeraldopenres.135 61.1

[7] C. Rollero, N. Piccoli, Does place attachment affect social well-being? European Review of Applied Psychology. No.60, pp. 233-238, 2010. https://doi:10.1016/j.erap.2010. 05.001

[8] L. Scannell, R. Gifford, Defining Place Attachment: A Tripartite Organizing Framework. Journal of Environmental Psychology. Vol. 30, No. 1, 2010. https://doi:10.1016/j.jenvp.2009.09.006

[9] E. Hall, The hidden dimension, New York, NY: Doubleday, 1966.

[10] J. Donohoe, The Place of Home. Environmental Philosophy, Vo. 8, No. 1, pp. 25-40, 2011.

[11] G. Bachelard, The poetics of space. Beacon Press, Boston, 1969.

[12] H., Coolen, J. Meesters, House, Home and Dwelling. Journal of Housing and the Built Environment, No. 26, pp. 1-10, 2012. https://doi:10. 1007/S10901-01 1-9247-4

[13] K., Lai, C. Webster, The nature of cities and the Covid-19 pandemic. Current Opinion in Environmental Sustainability, No. 46, pp. 27-31, 2020. https://doi.org/10.1016/j.cosust.2 020.08.008

[14] F. A. Ali, From Zoning Based Area to A Hybrid Space; The Transformation Strategies. JOURNAL OF Contemporary Urban Affairs, Vol. 3, No. 1, pp. 26-37, 2019. https://doi.org/10.25034/1764.ijcua3.1

[15] J. Faubion, Essential works of Foucault, 1954-1984. Vol. 2 , Aesthetics, method and epistemology. Penguin. 1998.

[16] G. Tokazhanov, A. Tleuken, M. Guney, A. Turkyilmaz, \& F. Karaca. How is COVID-19 Experience Transforming Sustainability Requirements of Residential Buildings? A Review. Sustainability, MDPI, Open Access Journal, Vol. 12, No.20, pp. 1-1, 2020. https://doi.org/10.3390/su122087 32.

[17] A. Ezezue, E. Ibem, C. Odum, B. Obiadi, Architectural Design Interventions for Sedentary Behaviour among Workers in Office Buildings in Enugu, Nigeria. Civil Engineering and Architecture, Vol. 8, No. 6, pp.1451 1462, 2020. https://doi.org/10.13189/cea.2020.080627.

[18] M. Pavlovic, An Experimental Approach to the Sophomore Architectural Design Studio. Civil Engineering and Architecture, Vol. 8, No.5, pp. 942-949, 2020. https://doi.org/ 10.13189/cea.2020.080521. 